\title{
Morpholino oligonucleotides do not participate perfectly in standard Watson-Crick complexes with RNA
}

\author{
GAOPING XIAO, ${ }^{1,3,4}$ DONNA WESOLOWSKI, ${ }^{1,3}$ MINA IZADJOO, ${ }^{2}$ and SIDNEY ALTMAN ${ }^{\mathbf{1}}$ \\ ${ }^{1}$ Department of Molecular, Cellular and Developmental Biology, Yale University, New Haven, Connecticut 06520, USA \\ ${ }^{2}$ Armed Forces Institute of Pathology, Washington, DC 20306-6000, USA
}

\begin{abstract}
RNase P from $E$. coli will cleave a RNA at a site designated in a complex with an external guide sequence (EGS). The location of the site is determined by the Watson-Crick complementary sequence that can be formed between the RNA and the EGS. Morpholino oligonucleotides (PMOs) that have the same base sequences as any particular EGS will not direct cleavage by RNase $P$ of the target RNA at the expected site in three mRNAs. Instead, cleavage occurs at a secondary site that does not correspond exactly to the expected Watson-Crick sequence in the PMO. This cleavage in the mRNA for a drug resistance gene, CAT mRNA, is at least second order in the concentration of the PMOs, but the mechanism is not understood yet and might be more complicated than a simple second-order reaction. EGSs and PMOs inhibit the reactions of each other effectively in a competitive fashion. A basic peptide attached to the PMO (PPMO) is more effective because of its binding properties to the mRNA as a substrate. However, a PMO is just as efficient as a PPMO on a mRNA that is mutated so that the canonical W-C site has been altered. The altered mRNA is not recognizable by effective extensive W-C pairing to an EGS or PMO. The complex of a PMO on a mutated mRNA as a substrate shows that the dimensions of the modified oligonucleotide cannot be the same as a naked piece of single-stranded RNA.
\end{abstract}

Keywords: RNase P; EGS; drug resistance; mRNA

\section{INTRODUCTION}

All specific biological interactions between two RNAs are governed primarily by Watson-Crick pairing between the molecules. These interactions, for example, determine what complexes are made between RNAs to form a substrate for the endoribonuclease RNase P. In fact, natural substrates are made by intramolecular pairing, but a non-natural substrate is made between two RNAs and is cleaved very efficiently by the enzyme. This latter process is involved in the external guide sequence (EGS) technology that can be used to inhibit the expression of genes in many organisms (Forster and Altman 1990).

At face value, the EGS technology offers great promise as a means of treating many different kinds of disease. However, there are significant problems in ensuring that one of the RNAs needed to form a complex cleavable by RNase $\mathrm{P}$ with a pathogenic RNA in vivo, the EGS, reaches with some

\footnotetext{
${ }^{3}$ These authors contributed equally to this work.

${ }^{4}$ Present address: Department of Pediatrics, Massachusetts General Hospital, Boston, MA 02114, USA.

Reprint requests to: Sidney Altman, Department of Molecular, Cellular and Developmental Biology, Yale University, New Haven, CT 06520, USA; e-mail: sidney.altman@yale.edu; fax: (203)432-5713.

Article published online ahead of print. Article and publication date are at http://www.rnajournal.org/cgi/doi/10.1261/rna.2256610.
}

efficiency the diseased site in humans. This problem, which is common with all RNA-based therapeutic means, can be partially solved by chemically modifying the RNA so it is resistant to any degradative ribonucleases found in extracellular media. A further step in this process that will yield modified oligonucleotides that will permeate cell types involves the covalent linkage of a basic peptide to one end of the modified oligonucleotide (Tilley et al. 2007; Mellbye et al. 2009).

Morpholino oligonucleotides (PMOs), which have been investigated by several groups, are resistant to degradative nucleases in extracellular serum (Summerton 1999). Other modified oligoribonucleotides that are relatively insensitive to degradative nucleases have also been developed recently (Soler Bistue et al. 2009). When the basic peptide, $(\mathrm{RXR})_{4} \mathrm{XB}$, is linked to one end of a $\mathrm{PMO}$, the hybrid molecule (called a PPMO) can permeate several bacterium (Mellbye et al. 2009). In pursuing this promising therapeutic lead, it has been found that the PMO or PPMO used to induce cleavage by RNase $\mathrm{P}$ in a specific pathogenic RNA in vitro or in vivo actually does not induce cleavage by RNase $\mathrm{P}$ at the expected site in the mRNA that is governed entirely by Watson-Crick interactions but at another site, perhaps $\geq 20$ nucleotides (nt) away from the theoretically expected site, as indicated below for three different genes, 
cat, gyrA, and $\operatorname{rnpA}$ (one asterisk shows a cleavage site for a PMO; three, a canonical cleavage site by an EGS) (Shen et al. 2009). A PMO appears to bind to the canonical "EGS"

- $c a t{ }^{41} \mathrm{CCAAU}^{\star}$ GGCAUGUAAAGAACAUUUU ${ }^{* *}$ GAGG CAUUUCAGUCAGUC

- $\operatorname{gyrA}{ }^{166} \mathrm{GGCAA}^{\star}$ GACUGGAACAAA. .52 nt...CCCA $\mathrm{U}^{\star * *}$ GGUGACUCGGCGGUUUGA

- $r n p A^{-23} \mathrm{UAAGU}^{\star}$ AAUAAAGCUGACCCCU ${ }^{\star * *}$ GAGUG GUUAAG

site, but the complex is not cleaved at that point but does get cleaved when it binds to a nearby site. The data indicate a need to examine the structural details of PMO and PPMO oligonucleotides to understand precisely how they bind to complementary RNAs. The same may be true of other modified oligonucleotides. Many of them seem to function in vivo as expected, but the actual mechanisms are unknown. Whether this anomaly is true just of binding a PMO to another RNA or is a characteristic of an altered substrate for RNase $\mathrm{P}$ remains to be shown.

\section{RESULTS}

\section{Kinetics with an mRNA-EGS substrate}

A measure of the kinetics of the reaction of RNase $\mathrm{P}$ and a CAT mRNA-EGS complex that has a susceptible site to RNase $\mathrm{P}$ is shown in Figure 1. In Figure 2, the data are plotted in terms of velocity (v) versus concentration of the particular oligonucleotide (EGS, PMO, and PPMO) used as a "substrate" (S). These kinetic data indicate that the reactions appear to be higher than first order for PMO and PPMO (Fig. 2B,C). There are lags in the plot of $\mathrm{v}$ versus [S], while that of an EGS is closer to first order (Fig. 2A). These data are confirmed in part as shown in Figure 3, in which the $\log$ of the velocity of the reaction is plotted versus the log of the concentration of the reactant, PMO (Fig. 3). The slope is 3.1 (see Cook and Cleland 2007) of the best fit straight line for the data, and the result is higher than a perfect second- order plot. The correlation coefficient is 0.97 , and the plot is one of three experiments. In the experiment in the graph shown, all the points were taken from one experiment, although more experiments were done with fewer experimental points. The control plots shows the graphs of the log of velocity versus the log of concentration of the PMO on the $\mathrm{EGS}^{*}$ substrate (slope is close to $1.5 \pm 0.1$ ), an mRNA that does not have the EGS site (see below), and on both the mRNAs with the wild-type (wt) $\mathrm{CAT}^{\mathrm{EGS}}$. These results, coupled with the anomalous site of cleavage of RNase $\mathrm{P}$ for PMO-mRNA and PPMO-mRNA that was reported earlier (Shen et al. 2009), are consistent with a reaction in which these latter two oligonucleotide-complexes as being higher than second order, that is, with respect to the number of EGS molecules involved in the reaction. The question of mechanism is discussed below. (The sequence of the PMO site in mRNA is the same as the PPMO-mRNA site: Small differences in gel mobility of the two complexes with PMO and PPMO can be ascribed to differences in the addition of the molecular weight of the peptide attached to the PPMO molecule; data not shown.) It remains to be shown if this means that one molecule of the PMO (or PPMO) is bound to the EGS site in mRNA but does not participate in the RNase $\mathrm{P}$ reaction before the reaction does take place at another site, or whether there are more complex phenomena involving enzyme reactivity in this process.

The inhibition of an EGS on the PMO (PPMO) reaction or the inhibition of a PMO (PPMO) on the EGS reaction is shown in Figure 4. The inhibition, given some uncertainty in the nature of the kinetics because of the nature of the "substrate," is competitive and complete (Taylor 2002; Cook and Cleland 2007; Bisswanger 2008). This result shows that the oligonucleotides bind to their respective cleavage sites freely in the mRNA and can be exchanged as such with other oligonucleotides in solution, although no binding constants have been measured here. These data with the PMO (PPMO) suggest that binding must occur to the EGS site first by these molecules before the PMO (PPMO) site is cleaved by RNase $\mathrm{P}$. We note also that at very high concentrations of the inhibitor, the velocity decreases. The precise explanation can
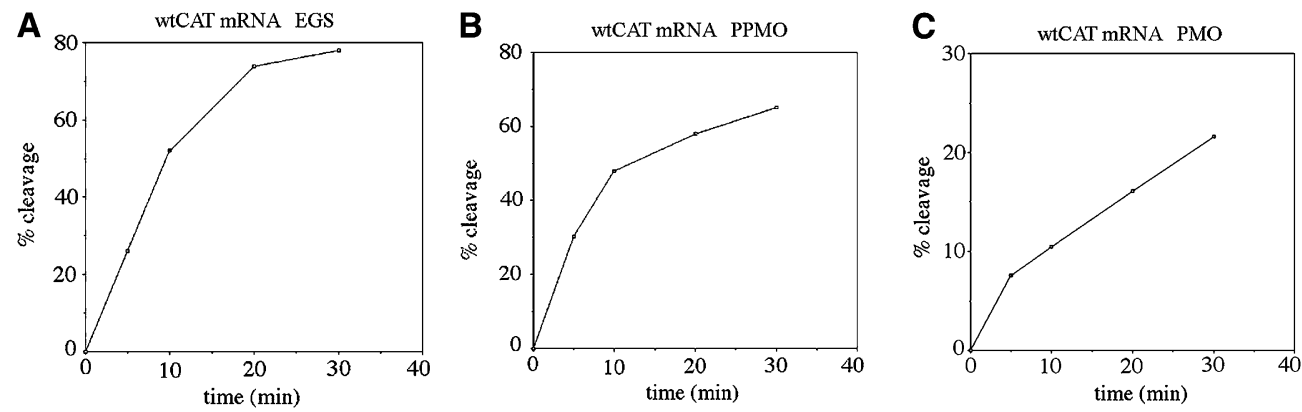

Figure 1. Amount of RNase $\mathrm{P}$ cleavage as a function of time. $(A) \mathrm{CAT}^{\mathrm{EGS}} ;(B) \mathrm{CAT}^{\mathrm{PPMO}} ;(C) \mathrm{CAT}^{\mathrm{PMO}}$. The methods for assay are in Materials and Methods. The amount of labeled mRNA (CAT mRNA) was $0.01 \mu \mathrm{M}$ and was used as substrate; EGS oligonucleotide, $0.5 \mu \mathrm{M}(50 \times)$; PPMO, $2 \mu \mathrm{M}(200 \times)$; and PPMO, $0.2 \mu \mathrm{M}(20 \times)$. At the top of this figure and the others, both the mRNA and the EGS used are listed as is appropriate. The experimental error is $\pm 5 \%$ for each point. 

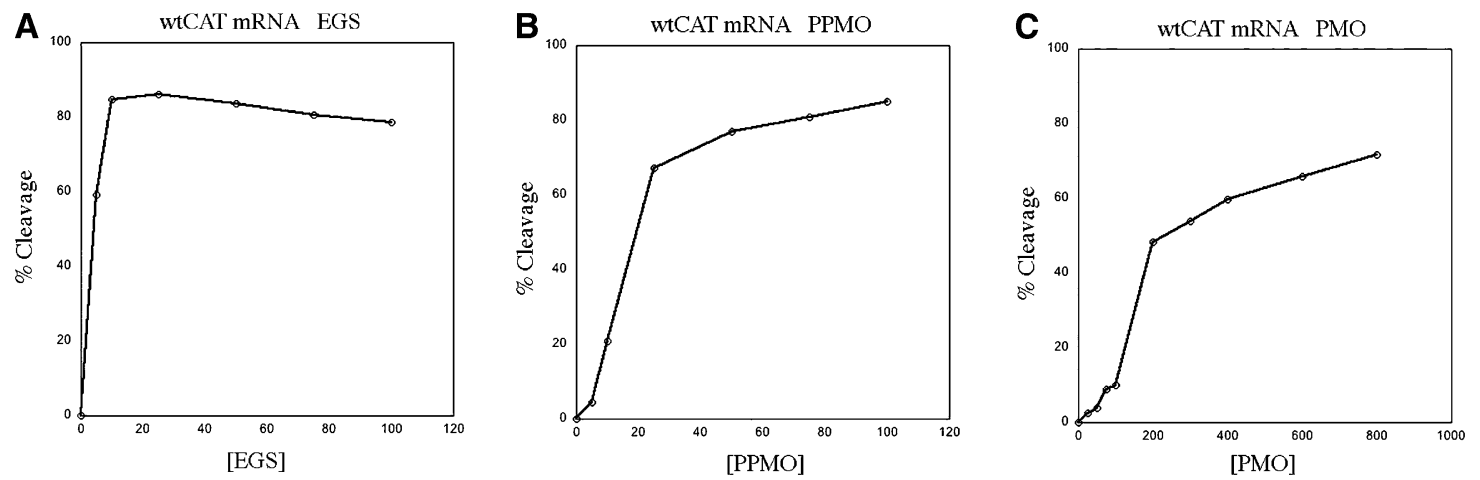

FIGURE 2. Order of reactions: Amount of cleavage as a function of concentration of the added EGS. $(A) \mathrm{CAT}^{\mathrm{EGS}} ;(B) \mathrm{CAT}^{\mathrm{PPMO}}$; $(C) \mathrm{CAT}^{\mathrm{PMO}}$. The concentration of added of the particular EGS oligonucleotide is given as a high ratio to the mRNA substrate as indicated in the abscissa of the figure.

be interpreted in several ways (Traut 2008), but we have not explored this phenomenon further.

It is not at all certain that a standard kinetic analysis is entirely accurate because of the fact that two molecules come together to form a complex that is the substrate, and this is discussed further below. Furthermore, the concentration of the enzyme used is not extremely small with respect to the concentration of the "substrate." The curves for PMO and PPMO indicate that inhibition occurs of the EGS at the EGS site (Fig. 4). It appears that inhibition is complete as judged by standard enzyme kinetics (Cook and Cleland 2007).

\section{The reactions with mutant mRNAs that lack either the EGS (EGS*) or the PMO (PPMO) site (PP*)}

The RNase $\mathrm{P}$ cleavage sites in the presence of EGSs and PMOs were altered so the sequences no longer resembled the wt sequences, as shown in the sequences written below (lowercase nucleotides are altered compared with the original site; see Materials and Methods; the PMO induced cleavage site is indicated by one asterisk in the CAT mRNA sequence, and the canonical cleavage site is indicated by three asterisks):

- CAT mRNA wt sequence- $-{ }^{41} \mathrm{CCCAAU}^{\star} \mathrm{GGCAUCGU}$ AAAGAA ${ }^{61}$ CAUUUU $^{* * *}$ GAGGCAUUUCAGUC ${ }^{80}$

- EGS or PMO (PPMO) - ${ }^{82}$ CTGACTGAAATGCCT ${ }^{66} \mathrm{CA}$ CCA 3'

- CAT mRNA EGS ${ }^{*}{ }^{61}$ CAUUUUucuuaAUUUCAGTC ${ }^{80}$

- CAT mRNA PP* — ${ }^{41}$ CCCAAUuuacgCGUAAAGAA ${ }^{60}$

The kinetic curves (Fig. 5) for CAT EGS* are simple and show no lag period near zero time for any of the oligonucleotides. In these cases, we do not expect any binding, of one kind or another, to the EGS site because it is absent. Thus, these results indicate a simple enzymatic reaction. The kinetics shown for the CAT EGS* mutation are also true for the curves on the CAT PP* mutant, as shown in Figure 5.

We note also that while there is no EGS site $\left(\mathrm{EGS}^{\star}\right)$, when the EGS oligonucleotide is employed in this reaction, there is a small, observable effect, about $20 \%$ of the normal rate of the PMO on the PP site, of cleavage by the EGS at the PP site.

The kinetics of inhibition (Fig. 6) on CAT EGS* and CAT $\mathrm{PP}^{\star}$ show the possible inhibition of the primary oligonucleotides PMO (PPMO) on the respective remaining sites. Inhibition is complete. Note that on CAT EGS*, there is no inhibition by PMO on the cleavage at the PMO site: This is simply part of the reaction. Varying the concentration of EGS in the EGS* case does show inhibition of PMO at the $\mathrm{PP}$ site, as seen in the figure. With the $\mathrm{PP}^{\star}$ mutant, PPMO has a clearly inhibitory function on cleavage by EGS at the EGS site, and PMO also does at a much higher concentration.

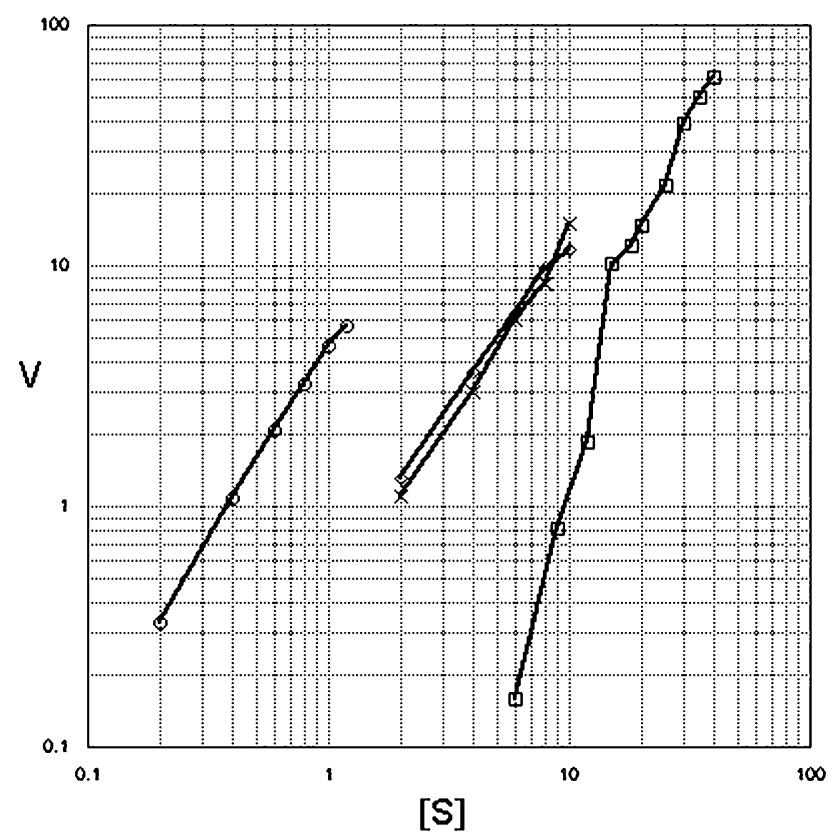

FIGURE 3. Kinetics of reactions. This is a log-log plot. The ordinate has the velocity of reactions, and the abscissa has the concentrations of the particular EGS added. The substrates are wildtype CAT mRNA and EGS ${ }^{\mathrm{CAT}}(\bigcirc)$, wild-type CAT mRNA and $\mathrm{CAT}^{\mathrm{PMO}}(\square), \mathrm{EGS}^{*} \mathrm{CAT} \mathrm{mRNA}$ and $\mathrm{CAT}^{\mathrm{EGS}}(\diamond)$, and $\mathrm{EGS}^{*} \mathrm{CAT}^{\mathrm{C}}$ mRNA and $\mathrm{CAT}^{\mathrm{PMO}}(\mathrm{X})$. 
A.

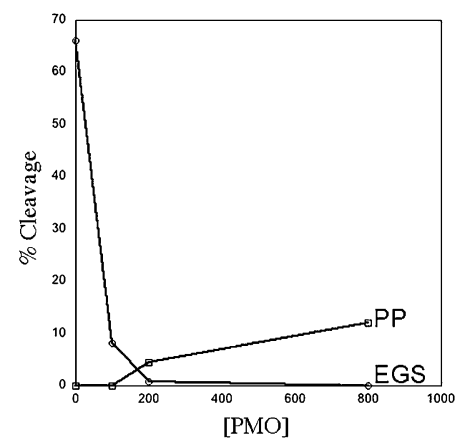

B.

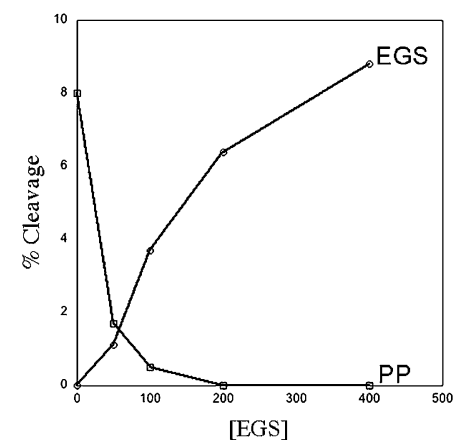

C. PPMO 10X, vary [EGS]

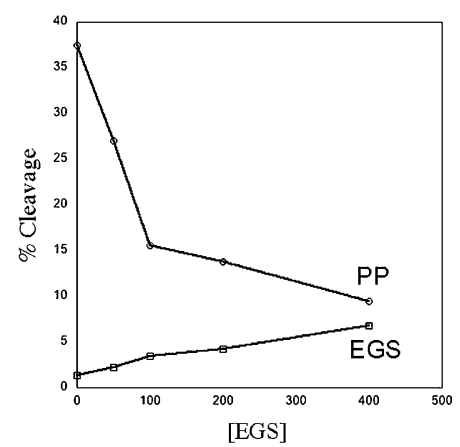

FIGURE 4. Inhibition by EGS, PMO, and PPMO on cleavage of wild-type CAT mRNA. (A) Cleavage of CAT mRNA substrate under competitive conditions. The amount of CAT ${ }^{\mathrm{EGS}}$ was constant at 50 times the amount of mRNA at the top of the graph. The amount of EGS ${ }^{\mathrm{PMO}}$ varied as indicated in the figure. Cleavage at the EGS or the PP site is indicated in the graph. (B) The amount of CAT ${ }^{\mathrm{PMO}}$ was constant, as shown at the top of the graph, and the amount of EGS varied. Cleavage is at different sites, as indicated in the graph. $(C)$ The same as in $B$ except the CAT ${ }^{\text {PPMO }}$ EGS $^{-1}$ was constant. Assay conditions are in Materials and Methods.

\section{Different substrates from CAT mRNA}

We cloned the $5^{\prime}$ end of the CAT mRNA in which the EGS site has been deleted; that is, the fragment cloned ended just downstream from the PP site and the CAT mRNA sequence ended at nt 60 (see Materials and Methods). Assays of RNase P cleavage with various EGSs and PPMOs, that is, a determination of the size of the fragments of cleavage and their sequences, indicated that cleavage occurred with the PPMO at the PP site as anticipated (data not shown), so primary binding to the EGS site obviously did not affect the reactions, as expected from the kinetics shown in Figure 6. With the reaction of the shortened CAT mRNA that only has the PP site, an additional, surprising cleavage site of RNase $\mathrm{P}$ alone in the absence of any EGS or PMO (PPMO) was also observed (data not shown). This cleavage, in the absence of any EGS or PMO or PPMO, was not at the PP site, was separable from the cleavages at the PP cleavage site, and was determined by a new secondary structure that produced a canonical cleavage site for RNase $\mathrm{P}$ in the short mRNA.

We also used the bla mRNA and the gyrA mRNA, both of which had been used previously and successfully with EGSs against their particular EGS site (Guerrier-Takada et al. 1997; Shen et al. 2009). These mRNAs were tried as controls in the current series of experiments. RNase P did not cleave the gyrA mRNA$\mathrm{EGS}^{\mathrm{CAT}}$ complex or the PPMO ${ }^{\mathrm{CAT}}$ or
PMO ${ }^{\text {CAT }}$ against gyrA mRNA. However, on bla mRNA, EGS $^{\text {CAT }}$ did not induce a cleavage by RNase P, but PPMO ${ }^{\text {CAT }}$ did work to a limited extent (the notation indicates a CAT PPMO and not a bla PPMO). An analysis of the possible hydrogen bonding of the PPMO ${ }^{\mathrm{CAT}}$ and $\mathrm{PMO}^{\mathrm{CAT}}$ to the bla mRNA indicate that a limited amount of pairing, $7 \mathrm{nt}$ in this
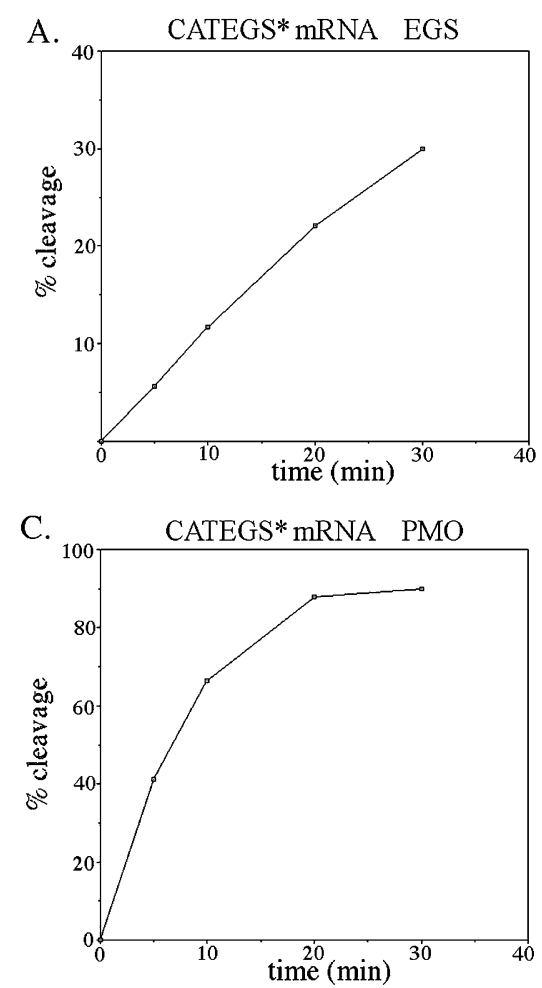

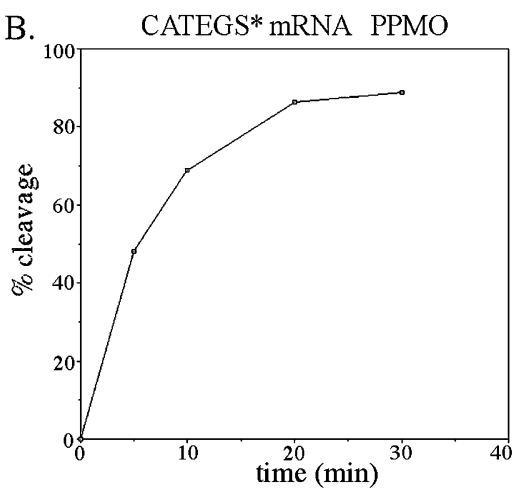

D.

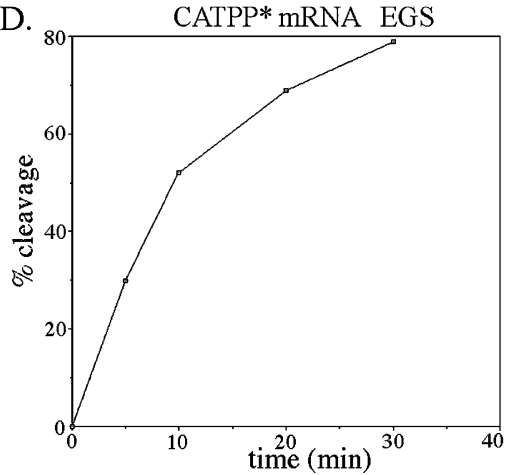

FIGURE 5. Cleavage assays for the $\mathrm{EGS}^{\star}$ and $\mathrm{PP}^{\star}$ mRNA mutants. The conditions are the same as for Figure 1 except the mutant substrates were used as indicated with wild-type EGS $(A), \operatorname{CAT}^{\text {PPMO }}(B)$, CAT $^{\text {PMO }}(C)$, and EGS $(D)$ used in the reactions. $(A-C)$ Substrate CAT EGS $^{*}$ (cleavage at the PP site). (D) Substrate CAT PP* (cleavage at the EGS site). The labels at the top of each graph indicate which substrate was used, and that is followed by which particular EGS was employed, as written in the legend to Figure 1. 

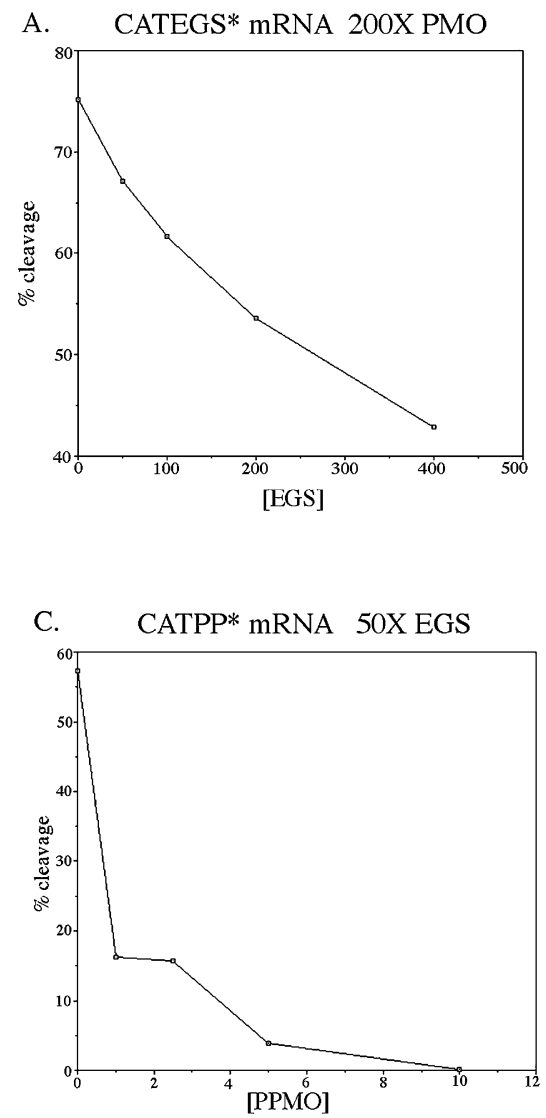

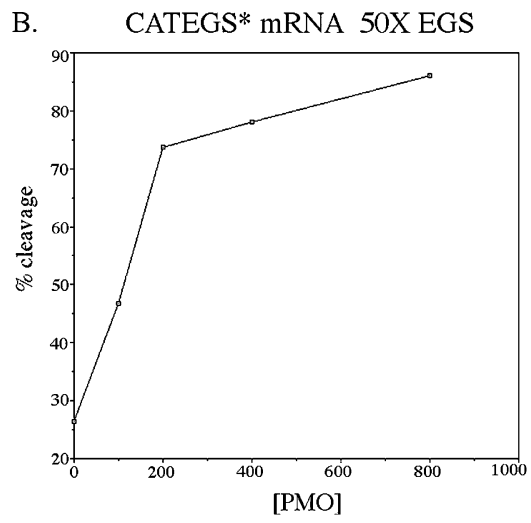

D. CATPP* mRNA 50X EGS

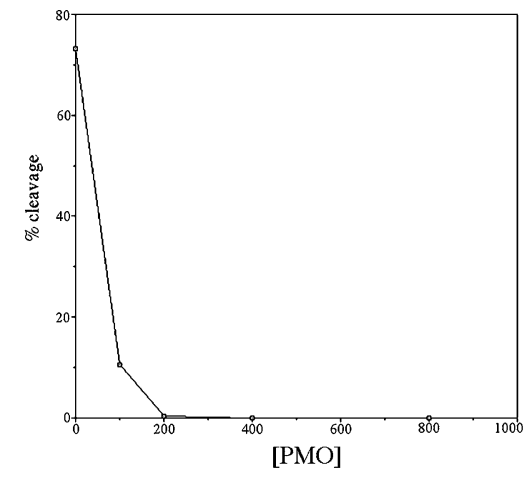

FIGURE 6. Inhibition of EGSs on CAT EGS* and CAT PP* mRNAs. Conditions were the same as used in Figure 4 except that the mRNAs were EGS $^{\star}$ and $\mathrm{PP}^{\star}$. Concentrations of particular EGSs are given in Figure 1. (A) EGS varied with CAT ${ }^{\mathrm{PMO}}$ held constant with EGS ${ }^{*}$ substrate. (B) CAT ${ }^{\mathrm{PMO}}$ varied and $\mathrm{CAT}^{\mathrm{EGS}}$ constant with CAT $\mathrm{EGS}^{*}$ substrate. $(C)$ EGS constant with $\mathrm{CAT}^{\mathrm{PPMO}}$ varied with $\mathrm{PP}^{\star}$ substrate. $(D) \mathrm{EGS}$ constant with $\mathrm{CAT}^{\mathrm{PMO}}$ varied with $\mathrm{PP}^{\star}$ substrate. $(A, B)$ Cleavage occurs at the PP site. $(C, D)$ Cleavage occurs at the EGS site.

case for a PMO (PPMO) EGS and the PP site, could occur, and is discussed further below:

- ${ }^{107}$ GGCAUUUUGCCUUCCUG-bla mRNA
CCGTAAAGTCAGTCAGTC-PMO

\section{The nature of mis-pairing of PMO with CAT mRNA}

The opportunity for PMOs and PPMOs to direct cleavage of the sequences in the CAT and bla mRNAs that were not fully complemented in a Watson-Crick sense to the PMOs oligonucleotides, but the impossibility of cleavage of a perfect WC base pair, indicated that the backbone dimensions of the PMO and PPMO were different from that of a naked single-stranded RNA or DNA. The fact that the theoretically possible introduction of bulges or small loops in a complex of two nucleic acids suggested that it might be possible to test the hypothesis with mutations in the EGS sequence in CAT mRNA. These alterations would especially allow a sequence in which the backbone is altered in comparison with the normal backbone of RNA. Accordingly, several deletions in CAT mRNA were made at the EGS and PP sites, and the resultant complexes with either the EGS or the PPMO nucleotides were checked for assay by RNase P. (One possibility is the PMO-mRNA complex would not fit into the active site of the enzyme at the EGS site but would at the PP site: This more complex arrangement would agree with our results but seems improbable.)

Several deletion mutations were introduced into a $\mathrm{PMO}^{\mathrm{CAT}}$ and into CAT mRNA (see Materials and Methods; Table 1). wt EGS ${ }^{\mathrm{CAT}}$ and $\mathrm{PMO}^{\mathrm{CAT}}$ were tested on different CAT mutant mRNAs in the presence of RNase P to see if any of the combinations produced cleavage of the mRNA. (Note that the relevant sequences are presented in the section concerning mutant mRNAs.) In fact, Table 1 shows that the wt PMO cleaves extremely well on the double deletion $\Delta \mathrm{G}_{70} \mathrm{C}_{71}$. In the presence of the deletion, $\Delta \mathrm{A}_{79}$, in a PMO EGS, cleavage by RNase $\mathrm{P}$ works well on the triple mutation $\Delta \mathrm{G}_{70} \mathrm{C}_{71} \mathrm{~T}_{79}$ (boxed numbers in Table 1 of deletions mentioned in the text). The deletion in the PMO EGS also worked well on the double deletion $\mathrm{G}_{70} \mathrm{C}_{71}$ in the substrate. The naked RNA EGS did not work on double- or triple-deletion mutants, as was expected from previous work on missense mutations (McKinney et al. 2001). In fact, no deletion mutations in the PMO EGS, except for the cases for the deletion in $\mathrm{A}_{79}$, worked on any of the deletion mutations in the CAT mRNA. However, the success with the deletion mentioned above in the PMO EGS and the deletions in the substrate indicate that the linear dimensions of the oligonucleotides must be important. These data do indicate that the dimensions of the PMOs create a shorter oligonucleotide than a naked RNA.

Cleavages that occurred at the EGS site but at a low level $(<25 \%)$ at the PP site were observed as one might expect by EGS $^{\mathrm{CAT}}$. The PPMO ${ }^{\mathrm{CAT}}$ cleaved the PP site but not the EGS site. (Two exceptions were $\Delta \mathrm{C}_{52}$ and $\Delta \mathrm{A}_{59} \mathrm{~A}_{60}$, which are located at the PP site. In these cases, little cleavage occurred at the PP site except for the wt PMO.)

While there are some obvious correlations, for example, triple, contiguous deletions drastically reduce cleavage as expected, there is no obvious alignment of the deletion mutations, that is, the nucleotides missing, with bases in the mRNA that would explain the cleavage results except for the cases cited. 
TABLE 1. Assays of RNase P induced cleavage in vitro of CAT mRNA and EGS (PMO) deletion mutations

\begin{tabular}{|c|c|c|c|c|c|c|c|c|}
\hline Substrate \EGS & Wild-type & $\mathrm{DC}_{70}$ & $\mathrm{DC}_{70} \mathrm{G}_{71}$ & $\mathrm{DA}_{79}$ & $\mathrm{DC}_{70} \mathrm{~A}_{79}$ & $\mathrm{DC}_{70} \mathrm{G}_{71} \mathrm{~A}_{79}$ & EGS & PPMO \\
\hline Wild-type CAT & 28.7 & 3.7 & 3.1 & 6.2 & 2.4 & 3.3 & 82.2 & 88.7 \\
\hline CAT DG $_{70}$ & 23.1 & 0 & 0 & 4.7 & 0 & 0 & 30.8 & 88.2 \\
\hline CAT $\mathrm{DG}_{70} \mathrm{C}_{71}$ & 91.4 & 5.0 & 0.3 & 75.3 & 3.9 & 0.1 & EGS/PPMO 29.7/24.3 & 93.9 \\
\hline CAT $\mathrm{DT}_{79}$ & 10.6 & 1.5 & 0.8 & 6.1 & 1.0 & 0.5 & 76.5 & 83.6 \\
\hline CAT D G ${ }_{70} T_{79}$ & 14.8 & 1.4 & 0 & 15.2 & 0.1 & 6.2 & 4.2 & 90.2 \\
\hline CAT $\mathrm{DG}_{70} \mathrm{C}_{71} \mathrm{~T}_{79}$ & 39.1 & 1.1 & 0.6 & 78.4 & 2.6 & 3.4 & 0.2 & 88.8 \\
\hline CAT $\mathrm{DT}_{79} \mathrm{C}_{80}$ & 6.2 & 1.5 & 1.7 & 4.6 & 0.9 & 0.8 & 74.2 & 88.7 \\
\hline CAT $\mathrm{DC}_{71} \mathrm{~A}_{72} \mathrm{~T}_{79}$ & 25.3 & 2.1 & 1.8 & 49.0 & 2.7 & 1.8 & 21.9 & 93.7 \\
\hline CAT PDC 52 & 48.5 & 2.7 & 18.2 & 12.5 & 0 & 0.4 & 88.8 & 93.9 \\
\hline CAT PDA $_{59} A_{60}$ & 12.1 & 7.3 & 1.3 & 7.8 & 1.0 & 1.1 & 88.4 & 78.1 \\
\hline CAT EGS* & & & & & & & $25 @$ PPMO site & 85 \\
\hline CAT PP* & & & & & & & 80 & 1 \\
\hline
\end{tabular}

The mRNA substrate is listed in the lefthand column, and the EGSs are listed across the top of the table. Boxed numbers are mentioned in the text. The first six EGSs are PMOs purchased as described in the Materials and Methods. The seventh column is an EGS made of naked RNA, and the last column is a PPMO EGS provided as a gift from AVI Biopharma (Materials and Methods). Assays were as described in the Materials and Methods. "D" refers to "deletion". In two cases, there was cleavage at an unexpected site, as listed. Most assays were done twice or more, especially those described in the text, but some were only done once. Errors in experimental values are $\pm 5 \%$.

\section{Chemical probing of complexes}

CAT mRNAs were labeled (see Materials and Methods; Regulski and Breaker 2008) and mixed with various EGSs and PMOs in standard buffers that contain divalent cations. The complexes were incubated for $40 \mathrm{~h}$ at room temperature and then analyzed on sequencing gels. The breakage of RNA occurs in single-stranded or exposed regions of RNA via chemical interactions between the divalent cations, the $2^{\prime} \mathrm{OH}$ and the phosphoester chain, and these breakage events can be visualized in a gel (Fig. 7). In complexes of an EGS, PMO, and a target RNA, the EGS site in the mRNA is protected (nt 67-82) (Fig. 7, lanes E, P under wt CAT and $\mathrm{CAT} \mathrm{PP}^{\star}$ ) and there is some cleavage in the PP site (nt 4762), but it is a minor variation compared with the EGS site. In the CAT EGS* lanes, there is cleavage with a somewhat different pattern at the now-mutated EGS site. The apparent, low amount of cleavage at the 47-62 site may be due to secondary or tertiary interactions in the mutated mRNA.

\section{DISCUSSION}

PMO or PPMO EGSs do not direct cleavage by RNase P of sequences in mRNAs that are perfectly complementary to their base sequences (Shen et al. 2009). This result is in direct contradiction of what is expected from normal considerations of WC base pairing and of data from studies of the EGS technology. Naked RNA, an EGS, behaves as expected in the experiments described here. However, cleavage by RNase $\mathrm{P}$ induced by a PMO (PPMO) did occur at base sequences $\sim 20 \mathrm{nt}$ from the oligonucleotide complex from the expected site (Shen et al. 2009). We note that there are $5 \mathrm{nt}$ in common in the sequences of the PP site and the EGS site in the CAT mRNA. These data compelled a more detailed analysis of the aberrant cleavage phenomenon.
The rough enzymatic kinetic analysis of the cleavage events indicated that the PMO-directed cleavage events that were higher than second order, while that of the RNA EGS was higher than first order. Each type of oligonucleotide, EGS versus PMO (PPMO) competed with the other in a complete, competitive reaction (Fig. 4).

One way in which the results can be interpreted assumes that the EGS, or PMO, binds to a site on the mRNA substrate, and there are two such sites on the substrate in the usual reaction. This might indicate that the equations for "second"-order kinetics can be described by an equation that satisfies the empirical data:

$$
2 \mathrm{~A}+\mathrm{M}(\mathrm{mRNA}) \rightleftarrows 3 \mathrm{~A}
$$

presuming that two sites are occupied and A is the EGS or PMO and M is the mRNA. The slope on a log-log scale for $\mathrm{v}$ versus $[\mathrm{S}]$ would be 3 if we also assume that the concentration of $\mathrm{M}$, the mRNA that contains two sites, is the same as the EGS. The "first"-order site the for the equation based on empirical observation is merely the first equation divided by two:

$$
\mathrm{A}+1 / 2 \mathrm{M} \rightleftarrows 1.5 \mathrm{~A}
$$

and in this case, the slope on a log-log scale of $\mathrm{v}$ versus [S] would be 1.5. These equations are correct in terms of fitting the data. However, the mechanism that provides these equations is not clear because of the binding of the different EGSs to the mRNA. There seems to be no folding of the mRNA itself to protect one site when an EGS is used, although we cannot be certain of this. More extensive experiments will be required to elucidate the mechanism.

A very simple interpretation of these results is that a PMO (PPMO) is bound to the EGS site, and another PMO (PPMO) induces cleavage rapidly at the PP site. There is a longer-lived interaction of the PMO with the EGS site and 


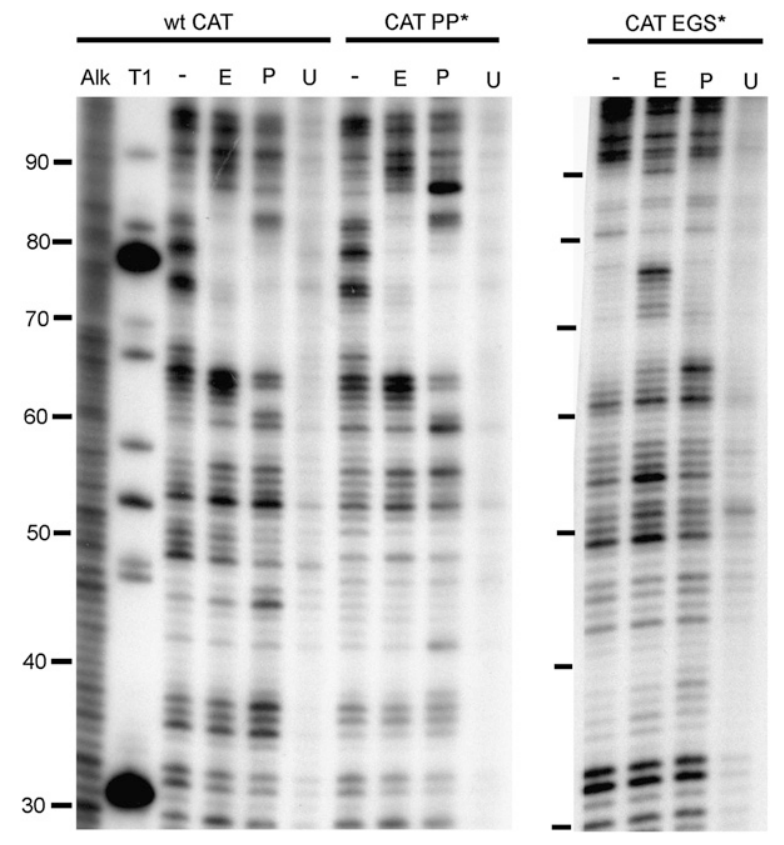

FIGURE 7. Chemical probing in-line experiments: Oligonucleotide complexes, in which the mRNA is labeled (Materials and Methods), were mixed and incubated as described in the Materials and Methods for $40 \mathrm{~h}$ at room temperature. The reactions were then mixed with a stop solution that contains $8 \mathrm{M}$ urea and dyes, analyzed in a $10 \%$ gel, and autoradiographed. RNA was labeled as described in the Materials and Methods. The figure shows the nucleotide size of markers on the lefthand side. The lanes are grouped under the mRNA that was used in the complex. Alk indicates alkaline digest of CAT mRNA; T1, partial RNase T1 digest of CAT mRNA; "-" no added EGS; E-wild type $\mathrm{CAT}^{\mathrm{EGS}}, \mathrm{P}^{\mathrm{C}} \mathrm{CAT}^{\mathrm{PMO}}$ EGS; and $\mathrm{U}$, unincubated mRNA.

a shorter one with the PP site, where cleavage of the mRNA occurs quickly. These hypotheses are supported by our inline experiments. Presumably, the dimensions of the PMO (PPMO) could not accommodate base pairing over $>5 \mathrm{nt}$, the distance that it might require for binding at the PP site and that can be identified in the gyrA and rnpA mRNAs. The rest of the PP site allows some pairing between individual bases that might aid in the binding to that site. A similar mechanism to the latter might allow cleavage of the bla mRNA, in which $7 \mathrm{nt}$ might be involved in pairing.

Deletion mutations in the CAT mRNA and in EGSs reveal that two or three contiguous deletion mutations in the mRNA reduce the level of cleavage by the wt PMO. One deletion mutation in the EGSs, A79, aids, to some extent, with the double and the triple deletions in the mRNA. These results are compatible with previous expectations (McKinney et al. 2001). No other strict correlation between the deletion mutations and the sequences in the mRNA and PMOs can be defined at the moment, but the linear dimensions of the PMO EGS must be different from a naked EGS. The PPMO EGS cleaves the PP site in all cases in which the deletions are at the EGS site. The flexibility of the backbone and base geometries of a PMO may not be identical to normal single- stranded DNA or RNA in a complex with another standard nucleic acid based on the known dimensions for A- or B-type helices (D. Crothers, personal communication). That would explain why only six or seven bases can participate in a $\mathrm{W}-\mathrm{C}$ complex with some distortion to accommodate all these particular bases. Subsequently, the dimensions of the PMO (PPMO) make it unable to interact perfectly in a WatsonCrick manner with the remainder of the paired oligonucleotide. This anomaly in the behavior of PMO and PPMO should be resolved by X-ray crystallography on the complexes of these oligonucleotides with fragments of mRNA that are perfectly complementary, that is, to the EGS site, or partly complementary, to the PP site. These proposed experiments will measure the exact dimensions of the PMOs under the seemingly appropriate conditions.

\section{MATERIALS AND METHODS}

\section{Bacterial plasmids}

The plasmids used for transcription were as described by Lundblad et al. (2008) and Shen et al. (2009).

For synthesis of mRNAs and EGSs, all mRNAs and EGSs were made as described by Guerrier-Takada et al. (1997), Lundblad et al. (2008), and Shen et al. (2009). The short CAT mRNA was transcribed from a product of the DNA used for transcription of CAT mRNA. It is terminated at $60 \mathrm{nt}$ in the cat gene.

\section{Mutants of mRNAs at the EGS and PP sites}

The Stratagene Quik Change site-directed mutagenesis kit and the method employed used the plasmid described above as template, and the method was employed to make altered EGS and PP sites: The primers for mutant mRNAs are as follows:

- $\mathrm{CAT} \mathrm{EGS}^{*}$ :

- CATE1: GGCATCGTAAAGAACATTTTTCTTAATTTCAGTC AGTTGCTCAAT

- CATE2: ATTGAGCAACTGACTGAaAtTAAgAAAAATGTTC TTTACGATGCC

- CAT PP*:

- CATPP1: CCGTTGATATATCCCAATTTACGCGTAAAGAAC ATTTTGAG

- CATPP2: CTCAAAATGTTCTTTACGCGTAAATTGGGATAT ATCAACGG

For synthesis of PMOs and PPMOs, the PMO oligonucleotides were purchased from GeneTools, and the PPMO oligonucleotides were a gift from AVI Biopharma.

\section{Assays of RNase P activity in vitro}

The assay was as described by Shen et al. (2009). Unless indicated otherwise, $0.01 \mu \mathrm{M}$ M1 RNA and $0.1 \mu \mathrm{M}$ C5 protein were used in the assays. The mRNAs used as substrates for cleavage assays were internally labeled by transcription in vitro using T7 RNA Polymerase (Promega) with $\left[{ }^{32} \mathrm{P}\right] \alpha$-GTP (Perkin Elmer). The labeled RNAs were purified on $5 \%$ polyacrylamide, $7 \mathrm{M}$ urea gels. 
In line cleavage

The protocol is listed by Regulski and Breaker (2008), and the reaction buffer contains $50 \mathrm{mM}$ Tris-HCI ( $\mathrm{pH}$ 8.3), $20 \mathrm{mM}$ $\mathrm{MgCI}_{2}$, and $100 \mathrm{mM} \mathrm{KCI}$. T7 RNA polymerase (Promega) was used to transcribe mRNA. The transcribed mRNA was dephosphorylated using rAPid Alkaline Phosphatase (Roche Applied Science), following the manufacturer's instructions, and then 5 ' end-labeled by T4 polynucleotide kinase (New England Biolabs) in the presence of $\left[{ }^{32} \mathrm{P}\right] \boldsymbol{\gamma}$-ATP (Perkin Elmer) and purified on a $5 \%$ polyacrylamide, $7 \mathrm{M}$ urea gel.

Although the efficiency of $5^{\prime}$ end-labeling PMO EGS with kinase is about 100-fold less than that of labeling mRNAs and the $3^{\prime}$ end labeling was 1000 -fold lower, there was no attempt to use these structures for the probing of chemical interactions or other experiments nor were the labeled structures characterized at all.

\section{Deletion mutations}

The method employed was the Stratagene Quik Change Site directed mutagenesis protocol as cited above. The following primers were used to introduce the mutations.

- CATDGF: GAACATTTTGAG-CATTTCAGTCAGTTGCTCAA TGTACC

- CATDGR: GGTACATTGAGCAACTGACTGAAATG-CTCAAA ATGTTC

- CATDGCF: GAACATTTTGAG-ATTTCAGTCAGTTGCTCAA TGTACC

- CATDGCR: GGTACATTGAGCAACTGACTGAAAT-CTCAAA ATGTTC

- CATDTF: GAACATTTTGAGGCATTTCAG-CAGTTGCTCAA TGTACC

- CATDTR: GGTACATTGAGCAACTG-CTGAAATGCCTCAAA ATGTTC

- CATDTG F: GAACATTTTGAG-CATTTCAG-CAGTTGCTCA ATGTACC

- CATdTGR: GgtACATTGAGCAACTG-CTGAAATG-CTCAA AATGTTC

- CATDTGCF: GAACATTTTGAG-ATTTCAG-CAGTTGCTCAA TGTACC

- CATDTGCR: GGTACATTGAGCAACTG-CTGAAAT-CTCAA AATGTTC

- CATDTCF: GAACATTTTGAGGCATTTCAG-AGTTGCTCA ATGTACC

- CATDTCR: GGTACATTGAGCAACT-CTGAAATGCCTCAAA ATGTTC

- CATDCATF: GAACATTTTGAGG- -TTTCAG-CAGTTGCTCA ATGTACC

- CATDCATR: GGTACATTGAGCAACTG-CTGAAA-CCTCAA AATGTTC

- CATPDCF: CCCAATGGCAT-GTAAAGAACATTTTGAGGCA TTTC

- CATPDCR: GAAATGCCTCAAAATGTTCTTTAC-ATGCCAT TGGG

- CATPDAAF: CCCAATGGCATCGTAAAG-CATTTTGAGGCA TTTC
- CATPDAAR: GAAATGCCTCAAAATG-CTTTACGATGCCAT TGGG

Once the mutated plasmids were verified via sequence analysis, mRNA was prepared using T7 transcription in vitro.

\section{ACKNOWLEDGMENTS}

G.X. and D.W. performed the experiments. G.X. and S.A. planned the project. S.A. wrote the manuscript. We thank members of our laboratory for helpful discussions and Drs. Ryzard Kole and Pat Iversen, AVI Biopharma (Corvallis, OR), for the provision of some morpholino oligonucleotides. This research was supported in part by grant W81 XWH-06-2-0066 to S.A. and M.I. and by the Armed Forces Institute of Pathology. The authors declare no conflict of interest.

Received May 6, 2010; accepted August 5, 2010.

\section{REFERENCES}

Bisswanger H. 2008. Enzyme kinetics: Principles and methods, 2nd ed. (trans. L Bubenheim). Wiley-VCH, New York.

Cook PF, Cleland WW. 2007. Enzyme kinetics and mechanism. Garland Science, New York.

Forster AC, Altman S. 1990. External guide sequences for an RNA enzyme. Science 249: 783-786.

Guerrier-Takada C, Salavati R, Altman S. 1997. Phenotypic conversion of drug resistant bacteria to drug sensitivity. Proc Natl Acad Sci 94: 8468-8472.

Lundblad EW, Xiao G, Ko JH, Altman S. 2008. Rapid selection of accessible and cleavable sites in RNA by Escherichia coli RNase P and random external guide sequences. Proc Natl Acad Sci 105: 2354-2357.

McKinney J, Guerrier-Takada C, Wesolowski D, Altman S. 2001. Inhibition of Escherichia coli viability by external guide sequences complementary to two essential genes. Proc Natl Acad Sci 98: 6605-6610.

Mellbye BL, Puckett SE, Tilley LD, Iversen PL, Geller BL. 2009. Variations in amino acid composition of antisense peptidephosphorodiamidate morpholino oligomer affect potency against Escherichia coli in vitro and in vivo. Antimicrob Agents Chemother 53: $525-530$.

Regulski EE, Breaker RR. 2008. In-line probing analysis of riboswitches. Methods Mol Biol 419: 53-67.

Shen N, Ko JH, Xiao G, Wesolowski D, Shan G, Geller B, Izadjoo M, Altman S. 2009. Inactivation of expression of several genes in a variety of bacterial species by EGS technology. Proc Natl Acad Sci 106: $8163-8168$.

Soler Bistue AJ, Martin FA, Vozza N, Ha H, Joaquin JC, Zorreguieta A, Tolmasky ME. 2009. Inhibition of aac $\left(6^{\prime}\right)$-lb-mediated amikacin resistance by nuclease resistant external guide sequences in bacteria. Proc Natl Acad Sci 106: 13230-13235.

Summerton J. 1999. Morpholino antisense oligomers: The case for an RNase H. independent structural type. Biochim Biophys Acta 1489: 141-158.

Taylor KB. 2002. Enzyme kinetics and mechanisms. Kluwer Academic, Boston.

Tilley LD, Mellbye BL, Puckett SE, Iversen PL, Geller BL. 2007. Antisense peptide-phosphorodiamidate morpholino oligomer conjugate: dose response in mice infected with Escherichia coli. J Antimicrob Chemother 59: 66-73.

Traut T. 2008. Allosteric regulatory enzymes. Springer, New York. 

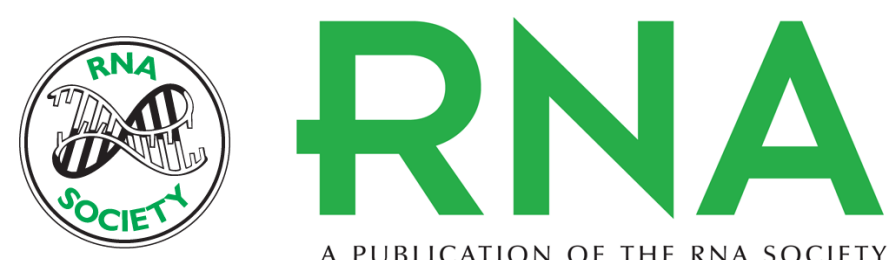

A PUBLICATION OF THE RNA SOCIETY

\section{Morpholino oligonucleotides do not participate perfectly in standard Watson-Crick complexes with RNA}

Gaoping Xiao, Donna Wesolowski, Mina Izadjoo, et al.

RNA 2010 16: 2218-2225 originally published online September 3, 2010

Access the most recent version at doi:10.1261/rna.2256610

\section{References This article cites 10 articles, 7 of which can be accessed free at: http://rnajournal.cshlp.org/content/16/11/2218.full.html\#ref-list-1}

License Email Alerting $\begin{aligned} & \text { Receive free email alerts when new articles cite this article - sign up in the box at the } \\ & \text { Service }\end{aligned}$ top right corner of the article or click here. 